\title{
Role of Technical Assistance in the Establishment and Scale Up of Programmatic Management of Drug Resistant Tuberculosis (PMDT) in Ethiopia
}

\author{
Ezra Shimeles $^{*}, 1$, Getachew Wondimagegn ${ }^{1}$, Ahmed Bedru ${ }^{1}$, Addisalem Yilma ${ }^{1}$, Dawit Assefa ${ }^{1}$, \\ Tesfaye Abicho ${ }^{1}$, Ermias Diro ${ }^{2}$, Yasmin Hashim ${ }^{3}$, Victor Ombeka ${ }^{4}$, René L’Herminez ${ }^{5}$ and \\ Eveline Klinkenberg,
}

${ }^{1} K N C V$ Tuberculosis Foundation, TBCARE I, Addis Ababa, Ethiopia

${ }^{2}$ Gondar University Hospital, Gondar, Ethiopia

${ }^{3}$ ALERT Center, Addis Ababa, Ethiopia

${ }^{4}$ KNCV Tuberculosis Foundation, Africa regional office, Nairobi, Kenya

${ }^{5}$ KNCV Tuberculosis Foundation, Central Office, The Hague, The Netherlands

${ }^{6}$ Department of Global Health, Academic Medical Center, University of Amsterdam, Amsterdam Institute for Global Health and Development, The Netherlands

\begin{abstract}
Implementation and scale up of programmatic management of drug resistant tuberculosis (PMDT) has proven to be a challenge in many resource-constrained settings like in Ethiopia, despite the political commitment.

A critical programmatic gap analysis was conducted by technical experts to develop feasible recommendations for incorporating multi drug resistant tuberculosis (MDR-TB) management in the national TB program following international guidelines and receiving continuous technical and financial support.

The major achievement was building of technical capacity in the country at the level of a national MDR-TB technical working group (TWG) as well as at the level of the MDR-TB treatment hospitals among the MDR management teams. In addition, guideline development, improved access to second line drugs and renovation of health facilities in accordance with TB infection control standards contributed to optimize implementation. Between February 2009 and September 2012, 575 MDR-TB patients accessed treatment under the program.

Building programmatic and clinical capacity at different levels of the health system in combination with strong political commitment and partner's engagement were key elements of the PMDT approach. Structured technical assistance combined with financial support during key steps was critical in the initiation and subsequent scale up of the program.
\end{abstract}

Keywords: Ethiopia, MDR-TB, PMDT, political commitment, technical assistance.

\section{INTRODUCTION}

Multi-drug resistant tuberculosis (MDR-TB) has become an emerging challenge for the TB control efforts in Ethiopia. The country ranks $8^{\text {th }}$ among the 22 global TB high burden countries (HBCs) and is also listed among the 27 global MDR-TB priority ${ }^{1}$ countries [1]. MDR-TB prevalence rate was $1.6 \%$ (95\% confidence interval [CI]: $0.90 \%-2.8 \%)$ among new TB patients and $11.8 \%$ (95\% CI: 5.6-21) among previous treated TB patients in the first nationwide anti-TB

*Address correspondence to this author at the KNCV Tuberculosis Foundation, TBCARE I, Addis Ababa, Ethiopia; Tel: +251 116632362; Fax: +251 116 632362; E-mail: shimelese@kncvtbc.nl

${ }^{1}$ The 27 high MDR-TB burden countries refer to those Member States estimated by WHO in 2008 to have had at least 4000 MDR-TB cases occurring annually and/or at least $10 \%$ of newly registered TB cases with MDR-TB. drug resistance survey (DRS) conducted in 2005 [2]. Several drug susceptibility testing (DST) methodologies for rifampicin and isonized have been introduced in the country since 2008. The process of expanding these DST services are confronted with many challenges and the coverage of DST among TB suspects is therefore still very low. In 2011 only $0.1 \%$ of new bacteriological confirmed TB patients and 3\% of previous treated TB patients had a DST result [3]. Latest diagnostic methods like Xpert MTB/RIF have been introduced in 2012 and are likely to expand. The proportion of retreatment cases that has been notified gradually increased from $1.3 \%$ in 1995 to $2.3 \%$ in 2010 and $3.1 \%$ in 2011 [4-6]. With the expansion of improved DST diagnostic services this is likely to continue to increase. A second DRS that started in 2012 can confirm if prevalence of drug resistance is also increasing. The latest global TB report estimates that Ethiopia has 1700 (range 910-2900) MDR-TB 
cases among new pulmonary and 440 (range 260-980) MDR-TB cases among pulmonary retreatment cases [5].

Being one of the 22 HBCs with a significant contribution to the global TB burden and a high estimated MDR-TB case load, several international organizations and donors, including USAID see Ethiopia as a priority country in the African Region. In July 2007, at the introduction of the USAID supported TB Control Assistance Program (TBCAP) in Ethiopia, a joint international technical assistance (TA) mission was conducted by technical staff from USAID and KNCV Tuberculosis Foundation (KNCV) to perform a situational analysis of the national TB program. This mission identified several challenges and potential opportunities for strengthening the TB control program and identified whether, and if so, where external support would be most required. The review provided key recommendations with respect to MDR-TB control indicating there is a need to: i) set up and build the capacity of a national health team to be involved in MDR-TB care and support; ii) build capacity for MDR-TB treatment in country, both in terms of facilities as well as programmatic capacity and iii) prepare a comprehensive strategic framework for a country-wide approach on MDR-TB. All these steps would require high level external technical support. The outcomes of the 2007 mission subsequently led to the launch of the USAID supported TBCAP program in Ethiopia starting in 2008.

\section{Technical Assistance}

The TB Coalition for Technical Assistance (TBCTA), with $\mathrm{KNCV}$ as the lead partner, is a unique coalition of the major international organizations in TB control. It consists of the International Union Against Tuberculosis and Lung Disease (the Union), the World Health Organization (WHO), Family Health International 360 (FHI360), Management Sciences for Health (MSH), American Thorax Society (ATS), and Japanese Anti-Tuberculosis Association (JATA). The coalition was established in 2000 as an answer to the global call for increased attention for tuberculosis. TBCAP was the second five year cooperative agreement (2005-2010) that was awarded to TBCTA. After completion of TBCAP, a third five year agreement (TBCARE I) was granted for the year 2010-2015, which has control of MDR-TB as one of its key objectives. As the lead partner of the TBCAP and TBCARE I programs in Ethiopia, KNCV was involved from the very beginning in the initiation of programmatic management of drug-resistant tuberculosis (PMDT) in the country, providing frequent and specialized international and national technical support to guide the roll out of the PMDT approach building on experiences gained in other countries. With the establishment of the KNCV office in Ethiopia in 2008 a strong base for in-country technical support was created. This has been instrumental in establishing and strengthening a national PMDT technical working group (TWG) and preparation of national policy guidelines and action plans on MDR-TB and infection control (IC). A key component of the contribution of the KNCV national technical staff is the continued support to human resource capacity building of staff of the National TB Program and involved local partners.

TBCAP's approach of providing technical assistance was primarily focussed on the identification of gaps and weaknesses and providing transparent and realistic recommendations to the attention of the national TWG. New activities were discussed, approved, implemented, and closely monitored with pro-active support and feedback by the local KNCV technical staff. Dedicated IC and PMDT focal persons were appointed within the TBCAP program to provide technical assistance and liaise with the Federal Ministry of Health $(\mathrm{FMOH})$ on the process of rolling out the activities. These focal persons were trained and received continuous guidance by KNCV international experts. Together with the National TB program management and other members of the TWG, identified gaps and challenges were discussed and targeted support to the health system was provided. The priority challenges identified to be addressed first were: i) the lack of a comprehensive strategic framework for a country-wide approach on MDR-TB; ii) the lack of human resource capacity as a result of both lack of experienced staff as well as high turnover of trained staff; iii) the lack of appropriate registration of second line drugs (SLDs) in the national drug list resulting in poor access to SLDs and iv) the limited hospital capacity for inpatient management of MDR-TB patients. These priority challenges were addressed in a systematic manner through on one hand, the development of a national MDR-TB strategy followed by a plan of activities and budget, which were both approved by the FMOH. And on the other hand, the mobilization of available technical and financial support and obtaining commitment of implementing partners to embrace and strengthen this outlined programmatic management of MDRTB approach in the country.

The global guidelines for the management of MDR-TB [7] provide a framework for DR-TB which is organized around the five components of the direct observed treatment therapy (DOTS) strategy: Sustained political commitment; a rational case-finding strategy including accurate, timely diagnosis through quality-assured culture and DST; appropriate treatment strategies that use second-line drugs under proper case management conditions; uninterrupted supply of quality-assured anti-tuberculosis drugs and a standardized recording and reporting system. This article describes what role technical assistance (TA) played in the establishment and scale up of PMDT in Ethiopia following the above outlined five key components.

\section{SUSTAINED POLITICAL COMMITMENT}

MDR-TB became a major concern for the FMOH of Ethiopia, after being sensitized on the global situation of the emergence of DR-TB, the findings of the national DRS, and the increasing number of confirmed DR-TB cases in the country. This situation urged the FMOH to put MDR-TB as a priority public health agenda which was clearly outlined in the five year strategic plan (2006-2010) which envisioned nationwide scale up of PMDT [8]. In 2006 the FMOH submitted a proposal for Global Fund round six with assistance of WHO and KNCV technical staff, assuring complementarities with other funding mechanisms. The proposal included a request to support MDR-TB components such as renovation of hospital wards to accommodate MDR patients, procurement of second line drugs, and activities addressing infection control including personal protective equipments like N-95 particulate respirator. The proposal aimed at an initial establishment of MDR diagnostic and 
treatment services at one central referral unit in the country. The granting of the proposal in the subsequent year initiated the start of PMDT in the country.

In April 2008, following USAID/KNCV recommendations, the FMOH established the MDR-TB TWG to provide overall guidance for the establishment of PMDT in Ethiopia. The group is composed of the FMOH and all major partner organizations that are involved and committed to support TB and MDR-TB control activities in the country. Its mandate includes the development of the national guideline for PMDT, a 5-year strategy and implementation plan for introduction and scale up of MDR-TB treatment services, development of curriculums for training of health care workers, and the design of a data management system. The TWG also developed recording and reporting formats for program monitoring tools. The first version of the national MDR-TB management guideline was completed in 2009 with assistance of external experts from several organizations that also provided overall guidance to further develop and strengthen the PMDT activities in the country. During several TA visits, the members of the TWG were oriented on the global perspective of scaling up PMDT and appropriate translation of international policy guidelines to national level policies. Members of the TWG and staff from the MDR hospitals attended overseas international training courses and joined experience sharing visits to initiatives in Cambodia, and Rwanda in the period of 2009-2012. This approach resulted in an increased practical in-country technical capacity in a relatively short time. All major partners represented in the TWG exchanged information on their contribution to the national implementation of MDRTB to ensure being complementary with other initiatives. The TWG provided the essential and strong foundation for the establishment of PMDT in Ethiopia.

\section{A RATIONAL CASE-FINDING STRATEGY INCLUDING ACCURATE, TIMELY DIAGNOSIS THROUGH QUA- LITY-ASSURED CULTURE AND DST}

An essential area for a successful MDR-TB program implementation is the strengthening of diagnostic capacity of drug resistant TB [7]. Conform to international WHO guidelines all previously treated confirmed TB cases should be tested for MDR-TB routinely using culture and DST. However due to insufficient laboratory capacity only $3 \%$ was tested in 2011 [5]. At that time there was only one culture and DST facility housed at the national TB reference laboratory in Addis Ababa. During the development of the PMDT program, additional culture diagnostic sites were identified. Assistance was provided for the establishment of new sites at the main MDR treatment centre, St Peters Hospital in Addis Ababa in 2011 and at two regional laboratories in 2012, Bahirdar in Amhara region and Adama in Oromia Region. Development of additional diagnostic capacity is anticipated during further scale up and should go hand in hand with the establishment of more treatment sites.

During the process of development and scale up of the PMDT program several challenges were encountered. The serious delay in the establishment of more diagnostic facilities hampered further patient enrolment. The issue was addressed by frequent discussions with stakeholders to stress the importance of improving diagnostic capacity. It was discussed in different national forums like the national laboratory technical working group meetings. Besides the limited quantitative and qualitative capacity of the national reference laboratory, that is leading the process of expanding the MDR-TB diagnostic network, the fact that the laboratory is answerable to another department within the FMOH than the National TB Program, made it difficult to influence the process.

Some more-complex laboratory investigations (e.g. hormonal, liver function tests) need to be done at the start and during treatment to enable appropriate monitoring of specific body functions and the conversion of the TB culture from positive to negative. To facilitate an early start of admission and management of MDR-TB patients at a new established treatment centre (ALERT centre) these essential tests were outsourced to a private laboratory.

The USAID support program TBCARE I provided technical and financial support for a national laboratory consultative workshop in March 2012 with participation of international experts and advisors in the field. Its objectives were to review and adjust the national laboratory strategy for both drug susceptible and drug resistant TB, address issues of external quality assurance (EQA) and review the current diagnostic algorithms. A follow up TA visit was organized to provide on the job training to improve skills of laboratory personnel on culture and DST as well as the Line probe assay. The first three GeneXpert MTB/RIF test machines were procured and handed over to the national program for the expansion of MDR-TB diagnostic service and the first training on GeneXpert MTB/RIF test for selected demonstration sites was supported as part of the national validation process, all in 2012. A plan for expansion of GeneXpert MTB/RIF is being developed and is expected to contribute to further acceleration of MDT-TB diagnosis.

\section{APPROPRIATE TREATMENT STRATEGIES THAT USE SECOND-LINE DRUGS UNDER PROPER CASE MANAGEMENT CONDITIONS}

Technical capacity on clinical management of MDR TB cases at all levels was created by development and implementation of training materials on drug resistant TB for the clinical teams in the health facilities as well as development of an in-country PMDT training. Orientation workshops were organized subsequently for the hospital staff which was meant to improve their general awareness for MDR-TB and TB IC. An experience sharing visit to the advanced program of Namibia in 2011 had a strong clinical focus. Besides training materials, other tools were developed including a Clinician's Reference Pocket on MDR-TB which serves as quick reference for management of MDR patients. Information, Education and Communication (IEC) materials (i.e., posters, leaflets, brochures) were developed to inform the public and patients about MDR-TB with key messages on adherence to TB treatment to prevent the development of $\mathrm{M}(\mathrm{X}) \mathrm{DR}-\mathrm{TB}$ and on household infection control measures.

In February 2009, the MDR-TB treatment program in Ethiopia was initiated as a pilot project at St. Peter Hospital in the capital city. The treatment comprised of a standard regimen of kanamycin, levofloxacin, cycloserine, ethionamide and para amino salycylic acid. Following the successful pilot in 2009 , the treatment program expanded to 
the northern part of Ethiopia at Gondar University Hospital in November 2010. Later, a third treatment centre was opened at the ALERT hospital also located in Addis Ababa in 2011. These sites were all renovated with support of several partners to make them suitable for MDR-TB treatment. The expansion of treatment sites reduced the waiting list from over 250 patients in 2009 to less than 100 at the beginning of 2012. The Ethiopia treatment policy requires admission of patients during the intensive phase for 4-8 weeks, to one of the three MDR hospitals. Patients are only discharged after sputum smear conversion. During the continuation phase, patients get their treatment from selected peripheral health facilities and take treatment under supervision of a treatment supporter. Despite all efforts to rapidly set up the services and increase access to diagnosis and treatment, there is still not enough admission capacity to take up the WHO estimated numbers of MDR-TB patients. To accelerate scale up of MDR treatment, the FMOH considers the introduction of the ambulatory model of care. Sites that will be selected for ambulatory care are those with minimum TB control requirements in place, which include well ventilated TB clinics, admission capacity for a small number of patients in case of side effects, staff trained on MDR-TB and TB IC as well as equipped with personal protective materials [9]. The ambulatory treatment program is planned to start implementation in 2013.

From February 2009 to September 2012 a total of 575 patients have been enrolled on treatment at the three MDR treatment sites. Of those the majority $(95 \%)$ were previously treated with first line drugs (FLDs), 2.5\% were previously treated with second line drugs (SLD) and 2.5\% were new cases that never received any anti-TB treatment. Nearly a quarter $(22 \%)$ of the resistant cases is co-infected with HIV. Two third (67\%) of these patients received ART during antiTB treatment as well. The majority of patients (98.6\%) are of the age groups $>15$ years. Of the 480 cases treated at $\mathrm{St}$ Peters $54 \%$ was male and $46 \%$ female.

The establishment of two additional treatment sites followed a standard approach of renovation of MDR-TB treatment wards, fully equipped with necessary supplies and equipment, establishment of data management unit, procurement of SLD as part of the national support, procurement of ancillary drugs and patient support through nutritional and psycho social support. A total of 130 beds were made available for MDR treatment between February 2009 and September 2012. Besides the renovation of wards, several TB IC materials (respirators, surgical masks, fans) were supplied and procurement and supply management of SLD and ancillary drugs was supported. In addition, support was provided for home visits, utilizing existing community support structures, to address IC at household level as well as adherence to treatment.

Further scale up is planned for the coming years including the renovating of MDR-TB treatment site for east Oromia region, renovation of outpatient department (OPD) complexes for ambulatory care model at several mainly peripheral centres, renovation of another culture and DST facility, and renovation of a MDR-TB training centre in Addis Ababa St. Peter hospital.

\section{UNINTERRUPTED SUPPLY OF QUALITY-ASSURED ANTI-TUBERCULOSIS DRUGS}

Ethiopia submitted a successful proposal to the Green Light committee (a global mechanism supporting scale up of PMDT including procurement of affordable quality SLD) in 2008 and got approval to start a pilot project. Procurement of SLDs through the GLC mechanism delayed but partners provided SLDs supporting a timely start of the pilot in 2009 at St. Peter Hospital in Addis Ababa. This was made possible through active engagement of the TWG with the assistance of international technical support. The technical support focused on developing the GLC proposal, selection and quantification of drugs, as well as support for the procurement process. During the process, local capacity on commodity management was strengthened. SLD procurement through GLC mainly funded through Global Fund and the USAID TBCARE I mechanisms increased from an initial 45 patients in 2009 to 600 in 2012.

From the start the MDR-TB drugs supplies management was handled by St Peter Hospital, for the three treatment sites, with technical support coming from several partners within the TWG. In 2013 the MDR treatment program drug supply management will be handed over to the integrated pharmaceutical logistic system as part of the national drug supply management system. This should align the management of SLDs with other TB commodities and supplies and avoid fragmentation of responsibilities.

\section{STANDARDIZED RECORDING AND REPORTING SYSTEM}

As part of PMDT scale up, a strong and good quality monitoring and evaluation (M\&E) system integrated into the national M\&E system to ensure proper management of data is needed. This will allow close monitoring and timely intervention in the complex movements between diagnostic and treatment services including the utilization of other patient support systems like psychosocial and nutritional support. The Ethiopian program developed an MDR-TB database using an MS Access platform. It was a result of consultative process by the TWG and health workers at the three MDR hospitals. External technical experts supported the consultative meetings and provided IT expertise. The user friendly database system captures all key information for MDR clinical management including standard patient information, clinical and laboratory data and treatment monitoring data. It automatically generates reports that are in line with the national MDR-TB reporting system requirements. The system became operational at the end of 2012.

Several partners provided TA to introduce and strengthen the recording and reporting at the TB treatment initiation centres through the establishment of a data management unit at each center occupied by a trained data manager provided with the necessary IT hardware and software.

To enhance M\&E, technical and financial support was provided for supportive supervision and mentoring of the hospital MDR teams as well as supportive supervision to the treatment follow up centers. TA was provided in developing supervision checklist and providing onsite training. TA also 
indentified gaps and provided hands on support to find and implement solutions. Besides supervision, PMDT specific regional review meetings and catchment area meetings to discuss progress in patient transfer and clinical management were supported.

Based on the recommendations for the enabling of optimal program monitoring, a car was donated to St Peter Hospital and Gondar University Hospital respectively, to support the logistics and coordination of MDR-TB activities in their catchment areas.

\section{DISCUSSION}

The establishment of a successful PMDT program in Ethiopia, with limited financial and human resources as inhibiting factors was in the first place realized through strong commitment of the FMOH and the National TB Program (NTP) that provided all necessary support to enable partners and donors to realize the proposed plans in collaboration with the NTP. The establishment and strong TWG with participation and strong commitment of all relevant partners have been of major importance. Join planning, open discussions and joint decisions on each other's role and responsibility resulted in an optimal use of the limited capacity and prevented overlap of contributions by the different partners. The continuous provision of international and local technical advice to the step wise approach and roll out of PMDT providing feedback and being pro-active was another crucial contribution. After developing the national PMDT guideline, the program slowly took root by putting in place the milestones required for ensuring the existence of functional PMDT in the country. Technical assistance helped the national program to develop national strategies on MDR-TB and TB-IC after which grants from the Global Fund and USAID were secured to support the implementation. Development of other relevant materials for strengthening the human resource and institutional capacity as a backbone of the program has been crucial as well. The collaboration of several partners within the TWG not only stimulated joint planning and budgeting resulting in complementary contribution by all major donors, it also created a joint feeling of responsibility.

The planned national scale up of PMDT will further increase patient recruitment which necessitates a continuous capacity building effort of health care staff, not just at the three hospitals but also at the follow up centers. To make a start to cover this national demand, TBCARE I conducted a series of trainings on PMDT with emphasis on early case detection and appropriate referral, to all health centers in Addis Ababa and to catchment health centers of Gondar MDR site. To further increase the number of MDR-TB confirmed patients for subsequent enrolment to the treatment program, the role out of GeneXpert, and strengthening of existing culture and DST laboratories will be crucial. Improved collaboration and focused support to the national TB reference laboratory will need to strengthen their capacity to take up the role as national coordinator of the laboratory expansion process adequately.

The critical role of TA is underlined by a program analysis of MDR-TB in three countries. Furin and colleagues [10] identify six overarching themes that need to be addressed in a successful MDR-TB program based on qualitative data collected over a 13 year period: 1) importance of baseline assessments, 2) early identification of key collaborators, 3 ) identification of initial locus of care, 4) minimization of patient-incurred costs, 5) targeted interventions for vulnerable populations and 6) importance of technical assistance and funding. The PMDT establishment and scale up in Ethiopia, followed most of these steps. It started by conducting baseline assessment identifying critical gaps, while the establishment of TWG matches with the early identification of key collaborators. St Peter hospital was selected as locus for piloting. Diagnostic and treatment services are provided free of charge to minimize patients cost. PMDT is initial targeting the group of MDR-TB patients diagnosed from screening previous treated patients. Technical assistance played an indispensable role throughout the whole process. The availability and provision of continuous local TA, for an important part guided by input from international experts, accepted and respected by all partners, is crucial for the implementation of the planned activities. Pro active feedback and interference, through discussions and joint decision making at the level of the TWG, provided continuous pressure on achieving the agreed targets.

\section{CONCLUSION}

In conclusion it is possible to improve access to MDR treatment in resource poor settings if there is strong government commitment and active engagement of key stakeholder in a partnership. Structural technical assistance during key steps in the initiation of the program and subsequent scale up are seen as essential for a successful implementation.

\section{CONFLICT OF INTEREST}

The authors confirm that this article content has no conflict of interest.

\section{ACKNOWLEDGEMENTS}

The authors wish to appreciate the strong government commitment and leadership by the FMOH of Ethiopia, dedication of the health care workers and active involvement of key partners to establish, implement and scale up a successful PMDT approach. The provided technical and financial support under TBCAP and TBCARE was made possible by the generous support of the American people through the United States Agency for International Development (USAID). The content of this article is the responsibility of the authors and does not necessarily reflect the views of USAID or the United States Government.

\section{REFERENCES}

[1] WHO. Towards universal access to diagnosis and treatment of multidrug-resistant and extensively drug-resistant tuberculosis by 2015. progress report 2011. Contract No.: WHO/HTM/TB/2011.3. Geneva, Switzerland: World Health Organization 2011.

[2] EHNRI: Report of the first National Ethiopian Drug Resistance Survey. Ethiopia: Addis Ababa 2005.

[3] Federal Ministry of Health. National TB Report, 201, Federal Ministry of Health. Ethiopia: Addis Ababa.

[4] Global Tuberculosis control. 2011 (WHO/HMT/TB/2011.16) Geneva, Switzerland: World Health Organization 2011.

[5] Global Tuberculosis control. 2012 (WHO/HMT/TB/2012.6) Geneva, Switzerland: World Health Organization 2012. 
[6] Global Tuberculosis control update 2009 (WHO/HTM/ TB/2009.426). Geneva, Switzerland: World Health Organization 2009.

[7] WHO. Guidelines for the programmatic management of drugresistant tuberculosis. WHO/HTM/TB/2008.402, 2008.

[8] Federal Ministry of Health. National strategic plan for TB control. Ethiopia: Addis Ababa.
[9] Federal Ministry of (2012). National protocol for ambulatory model of care. Ethiopia: Addis Ababa 2012.

[10] Furin J, Bayona J, Becerra M, et al. Programmatic management of multidrug-resistant tuberculosis: models from three countries Int $\mathbf{J}$ Tuberc Lung Dis 2011; 15(10): 1294-300.

Received: December 17, 2012

Revised: February 15, 2013

Accepted: February 17, 2013

(C) Shimeles et al.; Licensee Bentham Open.

This is an open access article licensed under the terms of the Creative Commons Attribution Non-Commercial License (http: //creativecommons.org/licenses/by$\mathrm{nc} / 3.0 /$ ) which permits unrestricted, non-commercial use, distribution and reproduction in any medium, provided the work is properly cited. 